\title{
Editorial: Collection in Memory of Mildred S. Dresselhaus
}

A year ago, on 20 February 2017, Mildred S. Dresselhaus, affectionately called "Millie," died at the age of 86, leaving behind an extraordinarily productive life in science. Scientists of all ages admired her untiring commitment to research and education until the very last minutes of her life. Encouraged by the managing editor of Physical Review Applied, Julie Kim-Zajonz, we decided to commemorate Millie's scientific legacy by a series of original articles related to her work on the pages of this journal.

It is very appropriate to publish such a collection in Physical Review Applied as Millie's research was highly interdisciplinary and had significant impact on many different fields in condensed matter and especially in applied physics. Her outstanding accomplishments in these areas have been rewarded with many prestigious awards, for example the Kavli Prize and the National Medal of Science in Engineering.

Much of Millie's life in science revolved around graphitic carbon, which earned her the title "Queen of Carbon Science." Starting in the 1960s with magneto-optic measurements of electron and hole states in graphite, Millie refocused her research interest onto graphite intercalation compounds and, in the late 1980s, onto carbon nanostructures. Of most impact was her development of resonance Raman spectroscopy into a powerful tool to characterize the properties and quality of nanocarbons and other related 2D materials. Her insight into optical and vibrational spectra of graphitic nanostructures provided improved understanding of the electron-phonon coupling and paved the way to a quantitative description of the fundamental band gap in nanotubes. Millie contributed maybe most significantly to the rapidly maturing field of carbon nanotubes, including the synthesis and characterization of doped nanotubes. With emerging interest in graphene monolayers, she provided new insight into the vibrational spectra of this $2 \mathrm{D}$ system and related graphene nanoribbons. Her expertise was guiding the scientific community as it changed its focus to recently discovered phosphorene as well as other 2D materials. Equally important was her most recent contribution to bismuth compounds used in thermoelectrics and to topological insulators. Her acquired knowledge has been condensed in around 1700 papers and books she authored, coauthored, and edited.

Millie's life in science was a journey of discovery. Again and again Millie took on the challenge to understand, interpret, and quantitatively describe new experimental observations. This gradual evolution process from discovery to interpretation, which then again leads to new discoveries, is the essence of science. Paying tribute to this evolutionary aspect of science, we will publish articles dedicated to Millie and her legacy not in a single issue, but in several issues throughout 2018.

The four papers included in this first issue published on the first anniversary of her death reflect Millie's view that fundamental and applied sciences can shine more brightly by mutual enrichment in a tight collaboration. The papers discuss ballistic and diffusive thermal conduction in graphene, water diffusion in carbon-based nanocomposites, the synthesis of enhanced-thermopower nanowire composites, and thermoelectricity in bulk and low-dimensional semiconductors.

We will complete the collection on her birthday in November. As guest editors, we feel that this is a very proper way to pay homage to Millie the Scientist and her legacy. We thank the many contributors to this collection for their support of this endeavor.

Morinobu Endo

David Tománek

Published 20 February 2018

Guest Editors

DOI: 10.1103/PhysRevApplied.9.020001 